\title{
Incremental cost-effectiveness of adult spinal deformity surgery: observed quality-adjusted life years with surgery compared with predicted quality-adjusted life years without surgery
}

\author{
Ian McCarthy, Ph.D., ${ }^{1}$ Michael O’Brien, M.D., ${ }^{2}$ Christopher Ames, M.D., ${ }^{3}$ \\ Chessie Robinson, M.A., ${ }^{1}$ Thomas Errico, M.D. ${ }^{4}$ David W. Polly JR., M.D., 5 \\ and Richard Hostin, M.D., ${ }^{2}$ on behalf of The International Spine Study Group
}

${ }^{1}$ Center for Clinical Effectiveness, Baylor Scott \& White Health, Dallas, Texas; ${ }^{2}$ Baylor Scoliosis Center, Plano, Texas; ${ }^{3}$ Department of Neurosurgery, University of California San Francisco, California; ${ }^{4}$ Department of Orthopaedic Surgery, NYU Hospital for Joint Diseases, New York, New York; and ${ }^{5}$ Department of Orthopaedic Surgery, University of Minnesota, Minneapolis, Minnesota

\begin{abstract}
Object. Incremental cost-effectiveness analysis is critical to the efficient allocation of health care resources; however, the incremental cost-effectiveness ratio (ICER) of surgical versus nonsurgical treatment for adult spinal deformity (ASD) has eluded the literature, due in part to inherent empirical difficulties when comparing surgical and nonsurgical patients. Using observed preoperative health-related quality of life (HRQOL) for patients who later underwent surgery, this study builds a statistical model to predict hypothetical quality-adjusted life years (QALYs) without surgical treatment. The analysis compares predicted QALYs to observed postoperative QALYs and forms the resulting ICER.

Methods. This was a single-center (Baylor Scoliosis Center) retrospective analysis of consecutive patients undergoing primary surgery for ASD. Total costs (expressed in 2010 dollars) incurred by the hospital for each episode of surgical care were collected from administrative data and QALYs were calculated from the 6-dimensional ShortForm Health Survey, each discounted at 3.5\% per year. Regression analysis was used to predict hypothetical QALYs without surgery based on preoperative longitudinal data for 124 crossover surgical patients with similar diagnoses, baseline HRQOL, age, and sex compared with the surgical cohort. Results were projected through 10-year follow-up, and the cost-effectiveness acceptability curve (CEAC) was estimated using nonparametric bootstrap methods.

Results. Three-year follow-up was available for $120(66 \%)$ of 181 eligible patients, who were predominantly female (89\%) with average age of 50. With discounting, total costs averaged $\$ 125,407$, including readmissions, with average QALYs of 1.93 at 3-year follow-up. Average QALYs without surgery were predicted to be 1.6 after 3 years. At 3- and 5-year follow-up, the ICER was $\$ 375,000$ and $\$ 198,000$, respectively. Projecting through 10-year followup, the ICER was $\$ 80,000$. The 10 -year CEAC revealed a $40 \%$ probability that the ICER was $\$ 80,000$ or less, a $90 \%$ probability that the ICER was $\$ 90,000$ or less, and a $100 \%$ probability that the ICER was less than $\$ 100,000$.

Conclusions. Based on the WHO's suggested upper threshold for cost-effectiveness ( 3 times per capita GDP, or $\$ 140,000$ in 2010 dollars), the analysis reveals that surgical treatment for ASD is cost-effective after a 10-year period based on predicted deterioration in HRQOL without surgery. The ICER well exceeds the WHO threshold at earlier follow-up intervals, highlighting the importance of the durability of surgical treatment in assessing the value of surgical intervention. Due to the study's methodology, the results are dependent on the predicted deterioration in HRQOL without surgery. As such, the results may not extend to patients whose HRQOL would remain steady without surgery. Future research should therefore pursue a direct comparison of QALYs for surgical and nonsurgical patients to better understand the cost-effectiveness of surgery for the average ASD patient.
\end{abstract}

(http://thejns.org/doi/abs/10.3171/2014.3.FOCUS1415)

\section{KeY WORDS • adult spinal deformity $\quad$ - scoliosis $\bullet \quad$ cost-effectiveness $\bullet$ health-related quality of life}

B ASED on the Nationwide Inpatient Sample, the total number of adult spinal deformity (ASD) surgeries more than doubled over the past decade, from

\footnotetext{
Abbreviations used in this paper: ASD = adult spinal deformity; $\mathrm{CEAC}=$ cost-effectiveness acceptability curve; HRQOL $=$ healthrelated quality of life; ICER = incremental cost-effectiveness ratio; QALY = quality-adjusted life year; SF-6D = 6-dimensional ShortForm Health Survey.
}

9400 in 2000 to more than 20,600 in 2010 (http://hcupus.ahrq.gov/nisoverview.jsp). This compares to just a $20 \%$ increase in the frequency of all other spine primary diagnosis codes over the same time period (from 675,500 in 2000 to 813,800 in 2010 ; http://hcup-us.ahrq.gov/nis overview.jsp). Adult spinal deformity surgery is likely to increase in frequency with as much as $32 \%$ of the adult population suffering from scoliosis and a prevalence of $60 \%$ among the elderly. ${ }^{18-21}$ 
In addition to increasing frequency, hospital costs of ASD surgery can easily exceed $\$ 100,000$ per patient after accounting for overhead allocation, readmissions, and complications following surgery. ${ }^{16}$ The real cost of treating adult scoliosis would be greater still, as these figures do not capture the large costs of outpatient care or prescription drugs, not to mention indirect costs incurred from lost productivity at work or family burden. As a result of increasing frequency and high costs of surgery, health care providers are under intense pressure to quantify the economic value of ASD and other complex spine surgeries. ${ }^{6,7,16}$

Yet despite the importance of cost-effectiveness studies in ASD, to date there have been no published studies attempting to directly measure the incremental cost-effectiveness of surgical treatment for ASD. Research in this area has been hampered not only by access to reliable cost data and validated, utility-based quality-of-life outcomes, but also by the inherent empirical difficulties in estimating the impact of surgical versus nonsurgical treatment. In particular, it is known that nonsurgical patients differ from surgical patients in meaningful ways, specifically with regard to baseline health-related quality of life (HRQOL). ${ }^{1,4,8,11}$ Given the ethical restrictions on randomized surgical/nonsurgical trials, identifying the accurate benefit of surgical treatment on quality-adjusted life years (QALYs) requires careful empirical consideration. ${ }^{13,14,17,25}$

The objective of this study was to assess the incremental cost-effectiveness of surgical versus nonsurgical treatment for ASD based on hypothetical predictions of QALYs for surgical patients as if surgery were never undertaken. This approach is intended to reflect the common argument that surgical treatment should be pursued while the patient can still recover from surgery and before the deformity increases and HRQOL further deteriorates. Intuitively, this argument may be most relevant for younger ASD patients, for whom treatment decisions are driven more by the deformity itself rather than degradation in quality of life..$^{111,22}$

\section{Methods}

This was a single-center retrospective study of consecutive ASD patients undergoing primary surgery with ICD-9 (International Classification of Diseases, Ninth Edition) principal diagnosis code 737.0-737.9 from January 2008 through June 2010 at Baylor Scoliosis Center in Plano, Texas. Patients younger than 18 years of age were excluded from the study. All patients underwent spinal fusion with a minimum of 3 levels fused.

Our measure of costs was the total costs incurred by the hospital for the episode of surgical care, expressed in 2010 dollars and totaled across the index surgery and any subsequent readmissions. Quality-adjusted life years were estimated from the 6-dimensional Short-Form Health Survey (SF-6D) and observed over a 3-year period, with HRQOL assessment at baseline (no more than 3 months prior to surgery for operative patients) and annually thereafter. ${ }^{2,3}$ Costs and QALYs were discounted at $3.5 \%$ annually. ${ }^{2,12}$

To assess the incremental effect of surgical treatment relative to nonsurgical treatment, a regression model was specified to predict hypothetical QALYs without surgery based on preoperative data. This model was estimated for a cohort of crossover surgical patients with similar diagnoses, baseline HRQOL, ages, and sex compared with the initial surgical cohort. This approach relies on data for patients with at least 2 preoperative HRQOL assessments who first visited the physician as nonoperative patients but ultimately underwent surgery after continued consultation.

Specifically, this approach estimates by ordinary least squares the following equation:

$$
\text { HRQOL }_{\mathrm{t}, \mathrm{i}}=\alpha+\mathrm{Z}_{\mathrm{i}} \beta_{1}+\text { HRQOL }_{\mathrm{t}-1 \mathrm{i}} \beta_{2}+\mathrm{Y}_{\mathrm{i}} \beta_{3}+\varepsilon_{\mathrm{t}, \mathrm{i}}
$$

in which HRQOL at time $\mathrm{t}$ is estimated as a function of HRQOL at time $\mathrm{t}-1$, along with the time elapsed (in years) from $\mathrm{t}-1$ to $\mathrm{t}$ and additional observed patient characteristics $(Z)$. The resulting coefficient estimates can be used to predict hypothetical HRQOL as if the patient had not undergone surgery, as a function of baseline HRQOL and other variables. Hypothetical QALYs without surgery were predicted 3 years into the future based on the regression model estimated in the equation above. Qualityadjusted life years through 5- and 10-year follow-up were then projected based on predicted QALYs as of year 3 .

The regression analysis provides an estimate of the incremental improvement in QALYs from surgery compared with hypothetical QALYs if surgery had not taken place. The ratio of average discounted hospital costs to the discounted improvement in QALYs yields the estimated incremental cost-effectiveness ratio (ICER). We then estimated the associated $95 \%$ CIs, as well as costeffectiveness acceptability curves (CEACs), using a nonparametric bootstrap technique with 5000 iterations. This technique iteratively samples, with replacement, individual costs and QALYs from the full data set. At each iteration we take the average costs of the bootstrap sample and estimate the incremental effect of surgery. The process yields a sample of 5000 costs and incremental QALYs from which to form the CEACs and 95\% CIs. ${ }^{24}$ All analyses were performed using Stata 12.1 (StataCorp LP), and institutional review board approval was obtained to conduct the study.

\section{Results}

This analysis relied on two separate cohorts of patients: 1) surgical patients with 3-year follow-up ( $\mathrm{n}=$ 120 of 181 consecutive patients with baseline HRQOL); and 2) crossover patients with two HRQOL assessments available preoperatively $(n=124)$. Table 1 summarizes the HRQOL outcomes and patient demographics for each cohort.

Regression analysis was used to predict hypothetical QALYs without surgery based on preoperative data for the 124 crossover surgical patients. Following the equation listed above, the regression specified follow-up HRQOL as a function of a patient's initial HRQOL, age, sex, interactions between these 3 variables, and the time between initial HRQOL assessment and follow-up (range 4 months to 2 years). These regression results are summarized in Table 2 . The results also satisfy a series of speci- 
Incremental cost-effectiveness of ASD

TABLE 1: Summary of HRQOL outcomes and patient demographics for each patient cohort

\begin{tabular}{lcc}
\hline \multicolumn{1}{c}{ Variable } & Surgical Group & Crossover Group \\
\hline female (\%) & 89 & 86 \\
mean age \pm SD $(\mathrm{yrs})$ & $50.531 \pm 13.20$ & $49.915 \pm 15.68$ \\
baseline HRQOL \pm SD & $0.620 \pm 0.12$ & $0.614 \pm 0.13$ \\
3-yr QALYs \pm SD & $1.931 \pm 0.34$ & - \\
no. of patients & 120 & 124 \\
\hline
\end{tabular}

fication tests, including the Shapiro-Wilk test of normally distributed errors $(p=0.28)$, the Ramsey RESET test of omitted variables $(p=0.50)$, and the link test of proper model specification $(\mathrm{p}=0.99)$.

We then applied the regression results from Table 2 to the observed data on the surgical cohort. Hypothetical QALYs were predicted through 1- and 2-year follow-up, and the percentage change from year 1 to year 2 was used to predict QALYs at years 3,5, and 10. These hypothetical QALYs were then compared with the observed QALYs at 3 -year follow-up, and the subsequent projections through 5- and 10-year follow-up, respectively.

The regression results in Table 2 predicted a hypothetical 1.60 QALYs without surgical treatment after 3-year follow-up, whereas an average of 1.93 QALYs were observed for this same patient group at 3 years following surgery (Table 1). Based on hospital costs of $\$ 125,407$, the ICER was estimated to be $\$ 374,428$ with a $95 \%$ CI of $\$ 311,400$ to $\$ 455,600$. Projecting through 5- and 10 year follow-up, the estimated ICERs were $\$ 198,000$ and $\$ 80,387$, respectively, with $95 \%$ CIs of $\$ 167,400$ to $\$ 237,000$ at 5-year follow-up and $\$ 69,800$ to $\$ 93,400$ at 10-year follow-up (Table 3).

The 10-year CEAC in Fig. 1 illustrates the probability that the dollar per QALY improvement falls below a given threshold value (the "willingness to pay" for surgical intervention). Each probability is calculated as the proportion of bootstrap-derived ICER estimates falling below a given cost-effectiveness threshold. For example, given a willingness-to-pay threshold of $\$ 90,000$ per

TABLE 2: Regression results for projected QALYs*

\begin{tabular}{lccc}
\hline \multicolumn{1}{c}{ Variable } & Coefficient & Standard Error & $\mathrm{p}$ Value \\
\hline initial HRQOL & 0.949 & 0.261 & 0.00 \\
age & 0.004 & 0.006 & 0.48 \\
female & 0.209 & 0.206 & 0.31 \\
time from initial survey & -0.024 & 0.021 & 0.25 \\
interaction terms & & & \\
age $\times$ initial HRQOL & -0.001 & 0.004 & 0.83 \\
female $\times$ initial HRQOL & -0.192 & 0.203 & 0.35 \\
age $\times$ female & -0.002 & 0.002 & 0.34 \\
constant & -0.089 & 0.260 & 0.73 \\
$\mathrm{R}^{2}$ & 0.582 & & \\
\hline
\end{tabular}

* Ordinary least-squares regression of 124 crossover surgical patients. Age squared was also included as a covariate in the model but was excluded from the table for brevity.
TABLE 3: Incremental cost-effectiveness ratios and $95 \% \mathrm{Cls}$

\begin{tabular}{lccc}
\hline \multicolumn{1}{c}{ Variable } & $3-Y r$ & $5-Y r$ & $10-Y r$ \\
\hline cost $(\$)$ & 125,407 & 125,407 & 125,407 \\
QALYs w/ surgery & 1.93 & 3.11 & 5.71 \\
QALYs w/o surgery & 1.60 & 2.47 & 4.15 \\
QALYs gained & 0.335 & 0.633 & 1.560 \\
ICER $(\$)$ & 374,428 & 198,000 & 80,387 \\
lower 95\% Cl (\$) & 311,400 & 167,400 & 69,800 \\
upper 95\% Cl (\$) & 455,600 & 237,000 & 93,400 \\
\hline
\end{tabular}

QALY gained, the CEAC estimates a 90\% probability that surgical intervention for ASD is cost-effective after 10-year follow-up provided HRQOL would deteriorate without surgery.

\section{Discussion}

Most of the cost-effectiveness studies currently available in the spine literature concern the cost-effectiveness of surgical treatment in the lumbar spine, including disc herniation and degenerative spondylolisthesis. For example, as part of the Spine Patient Outcomes Research Trial (SPORT), Tosteson et al. studied the cost-effectiveness of surgical versus nonsurgical treatment for lumbar disc herniation. ${ }^{23}$ The authors estimated costs based on patientreported utilization multiplied by Medicare per-unit allowable payment rates and measured outcomes as QALYs based on the EuroQol Group-5 Dimension (EQ-5D). The study reported an ICER of $\$ 69,403$ for all patients and an ICER of $\$ 34,355$ for Medicare patients. Based on direct costs only, the ICER increased to $\$ 72,181$ for all patients and \$37,285 for Medicare patients.

Glassman et al. analyzed the cost-effectiveness of single-level posterolateral lumbar fusion for 93 patients, with cost data based on actual reimbursements from third party payors and outcomes measured as QALYs based on the SF-6D., ${ }^{2,3,9}$ As part of the cost of surgery, the authors included outpatient visits up to 6 months after surgery. The authors reported an average reimbursement amount per QALY of $\$ 164,261$ after 1 year and $\$ 154,865$ after 2 years, although estimates varied depending on the patient cohort of interest.

Although baseline HRQOL is often cited as a major factor for surgical decision making, it is certainly possible that physicians look to additional, unobserved factors in deciding whether to discuss surgical options with patients. Our approach incorporates one such factor-namely, the belief that surgery should be pursued earlier while the patient can sufficiently recover and before HRQOL significantly deteriorates. As such, the underlying assumption is that the degradation in HRQOL for crossover patients would be the same, conditional on baseline HRQOL and other patient characteristics, for other patients who underwent surgery more quickly (that is, without a period of surveillance) and that this same degree of deterioration in HRQOL persists throughout the follow-up time frame. If our assumptions are not valid, and nonsurgical patients would have remained at their baseline HRQOL 


\section{McCarthy et al.}

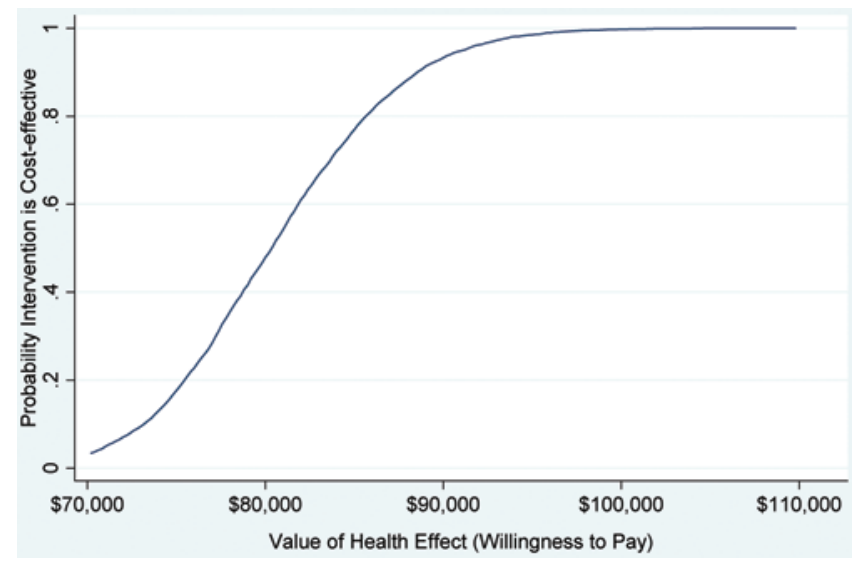

FIG. 1. Incremental CEAC of surgical treatment of ASD over a 10year follow-up.

values or improved in HRQOL over time, then prior work has shown that surgical treatment would not be as costeffective. In the absence of any deterioration, ongoing research has shown that ICERs for ASD surgery range from $\$ 276,000$ to $\$ 476,000 .^{15}$

Intuitively, several factors specific to spinal deformity likely influence the cost-effectiveness of ASD surgery compared with other spine studies in the literature. First, baseline HRQOL for ASD patients is higher on average than that of other spine surgery patients. For example, Glassman et al. reported a baseline SF-6D score of 0.5 and a postoperative SF-6D of 0.62 after 1 year. ${ }^{9}$ In our analysis, average SF-6D at baseline was 0.62 among surgical patients, with SF-6D scores after 1 year averaging 0.69 .

The high baseline HRQOL scores observed in our data are similar to those of other multicenter spine deformity studies. In particular, Bess et al., ${ }^{1}$ Bridwell et al., ${ }^{4}$ and Glassman et al..$^{10}$ report average baseline Oswestry Disability Index scores for surgical spine deformity patients of 33, 37 , and 34, respectively, much lower (lesser disability) than the average Oswestry Disability Index score of 56 reported in Glassman et al. ${ }^{9}$ in their 2012 study of single-level posterolateral lumbar fusion. Carreon et al. ${ }^{5}$ also reported an average baseline SF-6D of 0.53 among patients with scoliosis, which was the highest average baseline score among all diagnostic categories considered.

Second, our analysis includes HRQOL assessments for patients with readmissions subsequent to their primary surgery. For example, average QALYs after 3 years were 1.71 among patients with a readmission $(n=23)$, compared with an average of 1.98 among patients without a readmission. The total hospital costs for those with a readmission averaged $\$ 183,000$ compared with less than $\$ 112,000$ for patients without a readmission. Given the invasiveness and painful recovery following ASD surgery, a return to surgery reduces average QALYs while also adding to the average cost of surgery. Readmissions may therefore have a substantial effect on the overall ICERs, and hence on the estimated value of surgical treatment for the average ASD patient.

A final point concerns our measure of costs. In this analysis, we have considered only the hospital costs incurred for surgery and have not considered the cost of nonsurgical treatment. Our estimate of incremental costs is therefore taken solely as the direct costs of surgery incurred by the hospital, including overhead but excluding physician's fees. This exclusion is appropriate in a general sense, given that surgical costs are fundamentally incremental when compared with costs for nonsurgical patients; however, to the extent that nonsurgical costs incurred through physician visits, long-term physical therapy, or extended prescription drug usage differ from similar types of costs for surgical patients, our measure of incremental costs will not fully reflect differences in costs between the two groups. Over extended follow-up, the difference in costs between surgical and nonsurgical treatment may decrease in magnitude as nonsurgical treatment requires prolonged prescription drug usage, decreases productivity, and may even require surgical intervention at a later age. Conversely, surgical treatment costs may also increase over time due to future revisions or complications, but such increases would be relatively small on average. ${ }^{16}$ Additional indirect cost/benefit considerations associated with variation in productivity and absenteeism would further improve our analysis of surgical and nonsurgical treatment over an extended time frame.

\section{Conclusions}

Our analysis reveals two important findings with regard to the cost-effectiveness of surgical treatment for scoliosis and spinal deformity. First, the analysis estimates an ICER of $\$ 80,387$ per QALY gained from surgery for the average ASD patient over a 10-year followup. Based on the WHO's suggested upper threshold for cost-effectiveness ( 3 times per capita GDP, or $\$ 140,000$ in 2010 dollars), the analysis reveals that surgical treatment for ASD is cost-effective after a 10 -year period. These results represent a best-case scenario for an average ASD patient whose HRQOL would deteriorate under nonoperative care. At earlier follow-up intervals, the ICER well exceeds the WHO threshold, highlighting the importance of extended follow-up and accurate HRQOL prediction in the appropriate economic evaluation of surgical treatment for ASD.

From a policy standpoint, our findings may also support a brief period of surveillance for patients with relatively high baseline HRQOL. Provided we can identify patients whose HRQOL would further deteriorate without surgery, the results indicate that surgical treatment can be a cost-effective intervention for such patients. If deterioration in HRQOL is clearly documented prior to surgery, then the economic value of surgical intervention for such patients could be more easily supported and quantified.

\section{Acknowledgments}

We thank all the members of the International Spine Study Group and the attendees of the 2013 North American Spine Society and Scoliosis Research Society Meetings for their valuable comments and suggestions.

\section{Disclosure}

This study was based in part on funding from NuVasive, Inc., 
and the Baylor Health Care System Foundation, Seeger Endowment Fund. Dr. McCarthy has received a research grant from NuVasive Inc. Dr. O'Brien has served as a consultant to DePuy; has received royalties from Depuy, Medtronic, and Osteotech; and has received clinical or research support from DePuy for the study described. Dr. Ames has served as a consultant to DePuy, Medtronic, and Stryker; has direct stock ownership in Doctors Research Group, Visualase, and Baxano Surgical; is a patent holder for Fish \& Richardson, P.C.; and has received royalties from Aesculap and Biomet Spine. Dr. Errico has direct stock ownership in Fastenetix, has received support of non-study-related clinical or research effort from K2M, and has received grant support for research study and fellowship training from Paradigm Spine, OMEGA, OREF, AOSpine, and the Fridolin Trust. Dr. Hostin has received a research grant from NuVasive Inc.

Author contributions to the study and manuscript preparation include the following. Conception and design: McCarthy, O'Brien, Ames, Hostin. Acquisition of data: McCarthy. Analysis and interpretation of data: McCarthy, Robinson, Hostin. Drafting the article: McCarthy, Ames, Robinson. Critically revising the article: McCarthy, O'Brien, Ames, Errico, Polly, Hostin. Reviewed submitted version of manuscript: McCarthy, O’Brien, Ames, Errico, Polly, Hostin. Approved the final version of the manuscript on behalf of all authors: McCarthy. Statistical analysis: McCarthy, Robinson. Study supervision: O’Brien, Hostin.

\section{References}

1. Bess S, Boachie-Adjei O, Burton D, Cunningham M, Shaffrey C, Shelokov A, et al: Pain and disability determine treatment modality for older patients with adult scoliosis, while deformity guides treatment for younger patients. Spine (Phila Pa 1976) 34:2186-2190, 2009

2. Brazier J, Ratcliffe J, Tsuchiya A, Salmon J: Measuring and Valuing Health Benefits for Economic Evaluation. Oxford: Oxford University Press, 2007

3. Brazier J, Roberts J, Deverill M: The estimation of a preference-based measure of health from the SF-36. J Health Econ 21:271-292, 2002

4. Bridwell KH, Glassman S, Horton W, Shaffrey C, Schwab F, Zebala LP, et al: Does treatment (nonoperative and operative) improve the two-year quality of life in patients with adult symptomatic lumbar scoliosis: a prospective multicenter evidence-based medicine study. Spine (Phila Pa 1976) 34:21712178, 2009

5. Carreon LY, Djurasovic M, Canan CE, Burke LO, Glassman SD: SF-6D values stratified by specific diagnostic indication. Spine (Phila Pa 1976) 37:E804-E808, 2012

6. Deyo RA, Mirza SK: The case for restraint in spinal surgery: does quality management have a role to play? Eur Spine J 18 (Suppl 3):331-337, 2009

7. Deyo RA, Nachemson A, Mirza SK: Spinal-fusion surgerythe case for restraint. N Engl J Med 350:722-726, 2004

8. Glassman SD, Berven S, Kostuik J, Dimar JR, Horton WC, Bridwell K: Nonsurgical resource utilization in adult spinal deformity. Spine (Phila Pa 1976) 31:941-947, 2006

9. Glassman SD, Polly DW, Dimar JR, Carreon LY: The cost effectiveness of single-level instrumented posterolateral lumbar fusion at 5 years after surgery. Spine (Phila Pa 1976) 37:769774, 2012

10. Glassman SD, Schwab F, Bridwell KH, Shaffrey C, Horton W, $\mathrm{Hu}$ S: Do 1-year outcomes predict 2-year outcomes for adult deformity surgery? Spine J 9:317-322, 2009

11. Glassman SD, Schwab FJ, Bridwell KH, Ondra SL, Berven S, Lenke LG: The selection of operative versus nonoperative treatment in patients with adult scoliosis. Spine (Phila Pa 1976) 32:93-97, 2007
12. Gray AM, Clarke PM, Wolstenholme JL, Wordsworth S: Applied Methods of Cost-Effectiveness Analysis in Health Care. Oxford: Oxford University Press, 2011

13. Hoch JS, Briggs AH, Willan AR: Something old, something new, something borrowed, something blue: a framework for the marriage of health econometrics and cost-effectiveness analysis. Health Econ 11:415-430, 2002

14. Manca A, Hawkins N, Sculpher MJ: Estimating mean QALYs in trial-based cost-effectiveness analysis: the importance of controlling for baseline utility. Health Econ 14:487-496, 2005

15. McCarthy I, O’Brien M, Ames CP, Errico TJ, Kim HJ, Mundis GM Jr, et al: Cost-utility analysis of surgical treatment for adult spinal deformity. Spine J 13:S107-S108, 2013

16. McCarthy IM, Hostin RA, O'Brien MF, Fleming NS, Ogola G, Kudyakov R, et al: Analysis of the direct cost of surgery for four diagnostic categories of adult spinal deformity. Spine $\mathbf{J}$ 13:1843-1848, 2013

17. Nixon RM, Thompson SG: Methods for incorporating covariate adjustment, subgroup analysis and between-centre differences into cost-effectiveness evaluations. Health Econ 14:1217-1229, 2005

18. Robin GC, Span Y, Steinberg R, Makin M, Menczel J: Scoliosis in the elderly: a follow-up study. Spine (Phila Pa 1976) 7:355-359, 1982

19. Schwab F, Dubey A, Gamez L, El Fegoun AB, Hwang K, Pagala M, et al: Adult scoliosis: prevalence, SF-36, and nutritional parameters in an elderly volunteer population. Spine (Phila Pa 1976) 30:1082-1085, 2005

20. Schwab F, Dubey A, Pagala M, Gamez L, Farcy JP: Adult scoliosis: a health assessment analysis by SF-36. Spine (Phila Pa 1976) 28:602-606, 2003

21. Schwab FJ, Lafage V, Farcy JP, Bridwell KH, Glassman S, Shainline MR: Predicting outcome and complications in the surgical treatment of adult scoliosis. Spine (Phila Pa 1976) 33: 2243-2247, 2008

22. Smith JS, Shaffrey CI, Glassman SD, Berven SH, Schwab FJ, Hamill CL, et al: Risk-benefit assessment of surgery for adult scoliosis: an analysis based on patient age. Spine (Phila Pa 1976) 36:817-824, 2011

23. Tosteson AN, Tosteson TD, Lurie JD, Abdu W, Herkowitz $\mathrm{H}$, Andersson G, et al: Comparative effectiveness evidence from the spine patient outcomes research trial: surgical versus nonoperative care for spinal stenosis, degenerative spondylolisthesis, and intervertebral disc herniation. Spine (Phila Pa 1976) 36:2061-2068, 2011

24. Willan AR, Briggs AH: Statistical Analysis of Cost-Effectiveness Data. West Sussex, UK: John Wiley \& Sons, 2006

25. Willan AR, Briggs AH, Hoch JS: Regression methods for covariate adjustment and subgroup analysis for non-censored cost-effectiveness data. Health Econ 13:461-475, 2004

Manuscript submitted January 14, 2014.

Accepted March 14, 2014.

Portions of this work were presented in poster form at The Scoliosis Research Society Annual Meeting \& Course, Lyon, France, in September 2013, and in abstract form at The North American Spine Society 28th Annual Meeting, New Orleans, LA, in October 2013.

Please include this information when citing this paper: DOI: 10.3171/2014.3.FOCUS1415.

Address correspondence to: Ian McCarthy, Ph.D., Center for Clinical Effectiveness, Baylor Scott \& White Health, 8080 N. Central Expressway, Ste. 500, Dallas, TX 75206. email: ianmecarthy. econ@gmail.com. 